\title{
A DECISION SUPPORT SYSTEM FOR PLANNING AND CONTROLLING AGRICULTURAL PRODUCTION WITH A DECENTRALIZED MANAGEMENT STRUCTURE
}

\author{
Marek Makowski and Janusz Sosnowski \\ Systems Research Institute, Polish Academy of Sciences, Warsaw, Poland
}

\section{INTRODUCTION}

This paper presents some work done by the authors as part of a project aimed at the construction of a model of Polish agriculture [1] . The project has been undertaken by an interdisciplinary team composed of researchers from the Systems Research Institute, the Institute of Agricultural Economy, the Institute for Rural and Agricultural Development, in close cooperation with the state Planning Commission and the Ministry of Agriculture. The project represents a joint case study performed with the cooperation of the Food and Agriculture Program and the System and Decision Sciences Program of the International Institute for Applied Systems Analysis. Due to limitations on the size of the paper, only a brief outline is presented here. Details of the research may be found in [1], [2], [3].

\section{BACKGROUND AND STRUCTURE OF THE PROBLEM}

The institutional and technical context described below is crucial for understanding the problem.

Poland has a centrally planned economy. Therefore basic decisions (for example, on the annual socioeconomic plan, setting prices, interest rates, taxes, control of foreign trade) are made by central authorities forming the decision center (the Sejm (Parliament), Government, State Planning Commission, Ministry of Agriculture, the central committees of political parties and the main administrative institutions).

Polish agriculture has a diversified structure. It is composed of about 3 million units (farms) which are independent in their economic activity and self-managed. Over $70 \%$ of agricultural land is privately owned. Under the new economic system cooperative and state farms also enjoy independence in management.

The problem of direct versus indirect control of economic activities in a centrally planned economy within the context of Polish Agriculture is discussed in [1] and [4]. In current practice, profitoriented motives are used as the basic instrument for controlling 
agricultural production. The decision center is able to control both the production pattern and use of inputs more efficiently by setting prices and, if necessary, quotas than by any administrative measures (e.g. setting production targets).

Keeping in mind the context summarized above, we used the following approach to construct a decision support system for planning and control of agricultural production:

1. Producers are divided into 5 groups, each one (called a sector) being composed of producers with similar technological and behavioral characteristics. It is assumed that producers behave in a rational way, i.e. given the prices of all products and inputs, the technological constraints, and available inputs, the producers are assumed to choose a production plan for each sector which would maximize their own goal function. An LP model has been constructed and verified for each sector (see Sec. 3).

2. The agricultural production model ( $\mathrm{APM}$ ) is composed of sectoral models with appropriate linking conditions (see Sec. 4). The APM may be used by a decision center to assess the overall agricultural potential. In other words, it is possible, for assumed resources (inputs) and recognized overall goals, to determine a desired (and feasible) pattern and level of production and use of inputs for each sector.

3. Having decided on an overall plan, it is necessary to determine the economic instruments (mainly prices) that wauld enable parametric control of agricultural producers (see Sec. 5). The set of prices should ensure that while maximizing his own income each producer would choose a production pattern consistent with overall plan adopted by the decision center.

\section{MODELS OF SECTORAL PRODUCTION}

Keeping in mind the assumptions discussed in the previous section, we construct sectoral production models in the following form:

For each sector $i$ ( $i=1,2, \ldots 5$ ) find an activity level (production pattern and use of production inputs) $x_{i} \in R^{n}$ such that the producer's income is maximized:

$$
\begin{aligned}
& c \cdot x_{i} \rightarrow \max \\
& A_{i} x_{i} \leqslant b_{i} \\
& l_{i} \leqslant x_{i} \leqslant h_{i}
\end{aligned}
$$


where: c - given vector of prices for products and inputs,

$A_{i}$ - matrix of fixed coefficients for technical constraints,

$b_{i}$ - vector of available local inputs and resources (land, labour, etc).

Lower (if non-zero) and upper bounds for some activities may correspond to the behavioral characteristics of the producers. Lower bounds might be used for production targets for selected products, if required. Upper bounds may also reflect limits on available resources or quotas (see Sec. 5).

Sectoral models form the basis for constructing a set of admissible solutions for agricultural output as a whole. Therefore a lot of effort has been put into the construction and verification of those models by an interdisciplinary team.

\section{THE AGRICULTURAL PRODUCTION PLANNING MODEL}

Let us start by defining the admissible solution set for overall agricultural production. The set is defined by sectcral technical constraints together with linking constraints and may be written as:

$$
\begin{aligned}
& \mathrm{A}_{i} \mathrm{x}_{i} \leqslant \mathrm{~b}_{i} \quad i=1,2, \ldots 5 \\
& \sum_{i=1}^{5} \mathrm{~B}_{i} \mathrm{x}_{i} \leqslant \mathrm{~d}
\end{aligned}
$$

where constraints (5) correspond to limitations on the availability of production inputs for agriculture as a whole (the latter are defined by vector $d$, whereas the matrices $B_{i}$ are composed of elements which are equal to one or to zero). Appropriate constraints (3) are also included.

The structure of the admissible set for 3 sectors is illustrated in Figure 1.

Problems in agriculture are a specific feature of the current situation in Poland because of the imbalance between the supply of agricultural products and the demand for these goods. Food consumption accounts for nearly $60 \%$ of the consumers' income. The shortfall in the means of production (especially machinery and equipment, pesticides and concentrated feeds) stands in the way of increased agricultural production. Therefore, and due to the institutional context described in Sec. 2, it is hardly possible to define a single objective that could be used for the selection of a feasible solution for overall agricultural planning. Usually it is necessary to deal with several objectives, such as level of production of certain goods (others may be aggregated), level of use of selected inputs, balance of foreign trade 
in competitive agricultural products, and agricultural incomes. The set of criteria chosen (and its precise definition) depends on the user of the model and on the particular purpose to which the model is being put. Therefore a flexible software structure is necessary to allow easy definition of the criteria set and easy modification of the constraints set (selected variables may be either constrained or fixed in different runs).

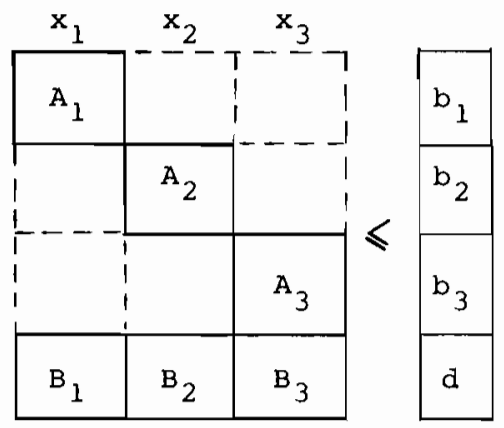

Figure 1. The structure of constraints for the overall problem.

The multicriteria nature of the problem makes it necessary to choose an appropriate methodology. After examination of all possible approaches the reference point (aspiration level) approach proposed by A. Wierzbicki (see [5]) has been adopted.

The aspiration level approach is now widely known, and therefore only a brief description will be given here. Let vector $\mathrm{q}$

$$
q=\sum_{i=1}^{5} Q_{i} x_{i}
$$

be a set of criteria (matrix $Q_{i}$ contains fixed weight coefficients). A decision center selects an initial aspiration level $\bar{q}$ (which may be attainable or non-attainable) and may attach weight coefficients to each criterion (weights not defined are assumed to be equal to one). With the help of an achievement function (see [7]) the problem is converted into the equivalent LP problem. The resulting solution is the non-dominated (Pareto-optimal) point, in criteria space, which is nearest (in the sense of a norm corresponding to a given achievement function) to $\bar{q}$ if $\bar{q}$ is nonattainable (i.e. if the aspiration level is too high) or is furthest from $\bar{q}$ if the aspiration level is too low. The corresponding decisions are computed at the same time. Having 
obtained a solution, the decision center may change an aspiration level and/or the weights attached to some criteria and repeat the procedure until an acceptable solution is found.

\section{CONTROLLING SECTORAL PRODUCTION}

The problem can be formulated as follows:

Having decided on an overall plan for agriculture, find instruments that make it possible to meet the following requirements:

1. The optimal sectoral plans (see Sec. 3) are consistent with the overall plan.

2. Prices (the same for all sectors) are the main instruments used to control sectoral planning.

3. Prices should fulfill additional constraints (resulting from the requirement that the level of sectoral income be within certain bounds, or to reflect some desired consistency within the price system, etc).

4. If there are no prices which fulfill requirements 1 through 3 , it is permissible to introduce a quota for products. If a quota is established, a fixed price is paid for a commodity only if the quantity sold does not exceed the quota. If there is a production surplus, a lower price may be paid. Constraints on inputs can also be introduced if necessary or used instead of quotas. However, the objective is to introduce as few quotas and/or limits as possible and - additionally there should be almost no reason for the violation of any such quotas or limits.

Let us first examine a situation in which a set of prices that fulfill requirements 1 through 3 exists. Assume that $\hat{x}_{i}, i=1,2, \ldots, 5$, is a solution of the APM and $\hat{A}_{i}$ are submatrices of $A_{i}$ composed of rows that were active in the solution considered. The conditions for optimality in $\hat{x}_{i}$ for sectoral problems can be formulated as follows:

$$
c-\hat{A}_{i}^{\mathrm{T}} \lambda_{i}-u_{i}=0 \quad i=1, \ldots, 5
$$

where: $c$ - is a vector of prices (positive components are for products, negative for inputs, zero for non-marketable commodities),

$\lambda_{i}$ - is a vector of Lagrange multipliers for active constraints in the $i$-th sector,

$u_{i}$ - is a vector of Lagrange multipliers for upper or lower bounds; therefore 


$$
\begin{array}{lll}
u_{i}^{j} \leqslant 0 & \text { if } & \hat{x}_{i}^{j}=l_{i}^{j} \\
u_{i}^{j}=0 & \text { if } & l_{i}^{j}<\hat{x}_{i}^{j}<h_{i}^{j} \\
u_{i}^{j} \geqslant 0 & \text { if } & \hat{x}_{i}^{j}=h_{i}^{j}
\end{array}
$$

where $j$ designates a particular product or input and $T$ means matrix transposition.

According to requirement number 3, additional constraints on prices may be formulated, for example:

$$
\begin{aligned}
& \underline{\underline{r}}_{i} \leqslant c \hat{x}_{i} \leqslant \bar{r}_{i} \\
& \underline{\underline{c}} \leqslant c \leqslant \bar{c}
\end{aligned}
$$

where $\underline{r}_{i}$ and $\bar{r}_{i}$ are lower and upper bounds on income in sector $i$, and $\stackrel{c}{=}$ and $\overline{\bar{c}}$ are lower and upper bounds on prices. It is assumed that $(* *)$ is feasible.

If the system of conditions (7), (*), (**) has a solution, it is usually non-unique. Therefore the following problem A can be formulated:

$$
\min \sum_{j=1}^{n} \frac{w 1}{c_{j}}\left|c_{j}-\bar{c}_{j}\right|
$$

subject to $(7),(*),(* *)$, where $\bar{c}$ is a vector of reference (desired) prices. If problem $A$ is feasible the set of prices that fulfill requirements 1 through 3 exists and can be determined.

It may happen that problem $A$ is infeasible. To illustrate a possible situation let us consider an example with two sectors and two commodities. Let the optimal solution of an overall plan (see Fig. 2) be $\hat{x}_{1}$ and $\hat{x}_{2}$ for sectors 1 and 2, respectively, and E1, E2, D1, D2 be active constraints.

A price vector has to be a linear combination of gradients of active constraints for each sector. Obviously no such price vector would be the same for both sectors. More detailed discussion of this problem may be found in [2] or in [3].

If the problem $A$ is infeasible one may formulate a partially perturbed problem $B$ as follows:

$$
\min \left(\sum_{j=1}^{n} \frac{w 1}{c_{j}}\left|c_{j}-\bar{c}_{j}\right|+w 2 \sum_{i=1}^{5} \sum_{j=1}^{n} v_{i}^{j}\right)
$$


subject to

$$
\begin{aligned}
& c-\hat{A}_{i}^{T} \lambda_{i}-u_{i}-v_{i}=0 \quad i=1, \ldots, 5 \\
& v_{i} \geqslant 0
\end{aligned}
$$

and $(*),(* *)$.

The non-zero (if any) eomponents of vectors $v_{i}$ correspond to products for which quotas are allowed and to inputs for which limits are permitted (the concept of quotas has been proposed by A.P. Wierzbicki [8]). One may also add conditions for quota shadow prices (see [2]). If the problem $B$ is feasible a set of prices can be determined. Note that $v_{i}^{j}>0$ implies the introduction of a constraint on commodity $j$ in sector $i$ of the type $x_{i}^{j} \leqslant \hat{x}_{i}^{j}$ (where $\hat{x}_{i}^{j}$ is a component of the solution of the overall plan), $i . e$. introduction of either a quota or a limit (depending on whether the variable is a product or an input).
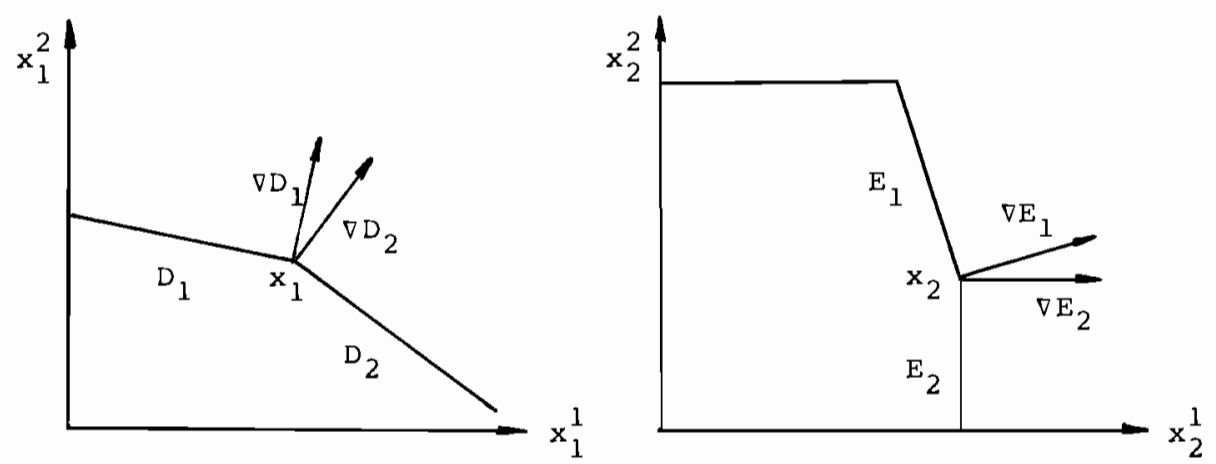

Figure 2. A case for which a common set of prices does not exist.

Should the problem $B$ be infeasible (which may be caused by "tight" conditions $(* *))$ the following perturbed problem $C$ may be formulated:

$$
\min \left(\sum_{j=1}^{n} \frac{w 1}{c_{j}}\left|c_{j}-\bar{c}_{j}\right|+\sum_{i=1}^{5} \sum_{j=1}^{n}\left(w 2 \cdot v_{i}^{j}+w 3\left|t_{i}^{j}\right|\right)\right)
$$

subject to

$$
\begin{aligned}
& c-\hat{A}_{i}^{\mathrm{T}} \lambda_{i}-u_{i}-v_{i}-t_{i}=0 \\
& v_{i} \geqslant 0
\end{aligned}
$$

and $(*),(* *)$.

It is easy to see that problem $C$ is always feasible. However, its 
solution does not satisfy requirement number 1 . If the values of vectors $t_{i}$ are small one may expect that the solutions of the sectoral problems would not differ much from those of the chosen overali plan. Therefore the sectoral problems have to be solved and the solutions examined to check whether the differences are acceptable. If the resulting sectoral solutions are not acceptable either some conditions on the prices have to be slackened or another overall plan should be examined.

The weight coefficients $w 1$, w2 and $w 3$ reflect the preferences of the decision center. Usually w3 has the biggest value because the consistency of sectoral and overall plans is generally of greatest importance. If it is preferred to have the price structure "closer" to the desired structure, at the expense of introducing more quotas or limits, then 11 should be greater than $w 2$. In the opposite situation the relation should be reversed.

\section{THE STRUCTURE OF THE DECISION SUPPORT SYSTEM}

The decision support system is composed of a system of programs implemented on a UNIVAC 1100 at the Computation Centre of the state Planning Commission in Warsaw. The software may be used in different ways in order to meet the specific needs of particular users (see [9]).

The typical mode of operation of the decision support system is presented in Figure 3 .

One starts with sectoral models, which are generated using a database according to the current requirements of the decision center. Then a set of requirements and sectoral models are used to generate a multiobjective planning problem covering the whole agricultural system. This problem is solved severaltimes (for different assumptions concerning aspiration levels, constraints, etc.) until an acceptable solution is found. Then the next set of decisions on reference prices, quota conditions, etc.) is used to generate the price problem, which is also solved several times until an acceptable solution is found. It may then be necessary to re-examine sectoral problems if Problem $\mathrm{C}$ is being considered (see Sec. 5).

At each stage of computation, reports containing information necessary for the evaluation of a solution by the decision maker are produced. Programs that enable easy generation of new scenarios have also been developed.

The software allows efficient analysis of the problems described in this paper. However, for the time being at least, it is not a fully "automatic" system, and therefore it is necessary to contact one of the designers of the model for information on some parts of the computations. 


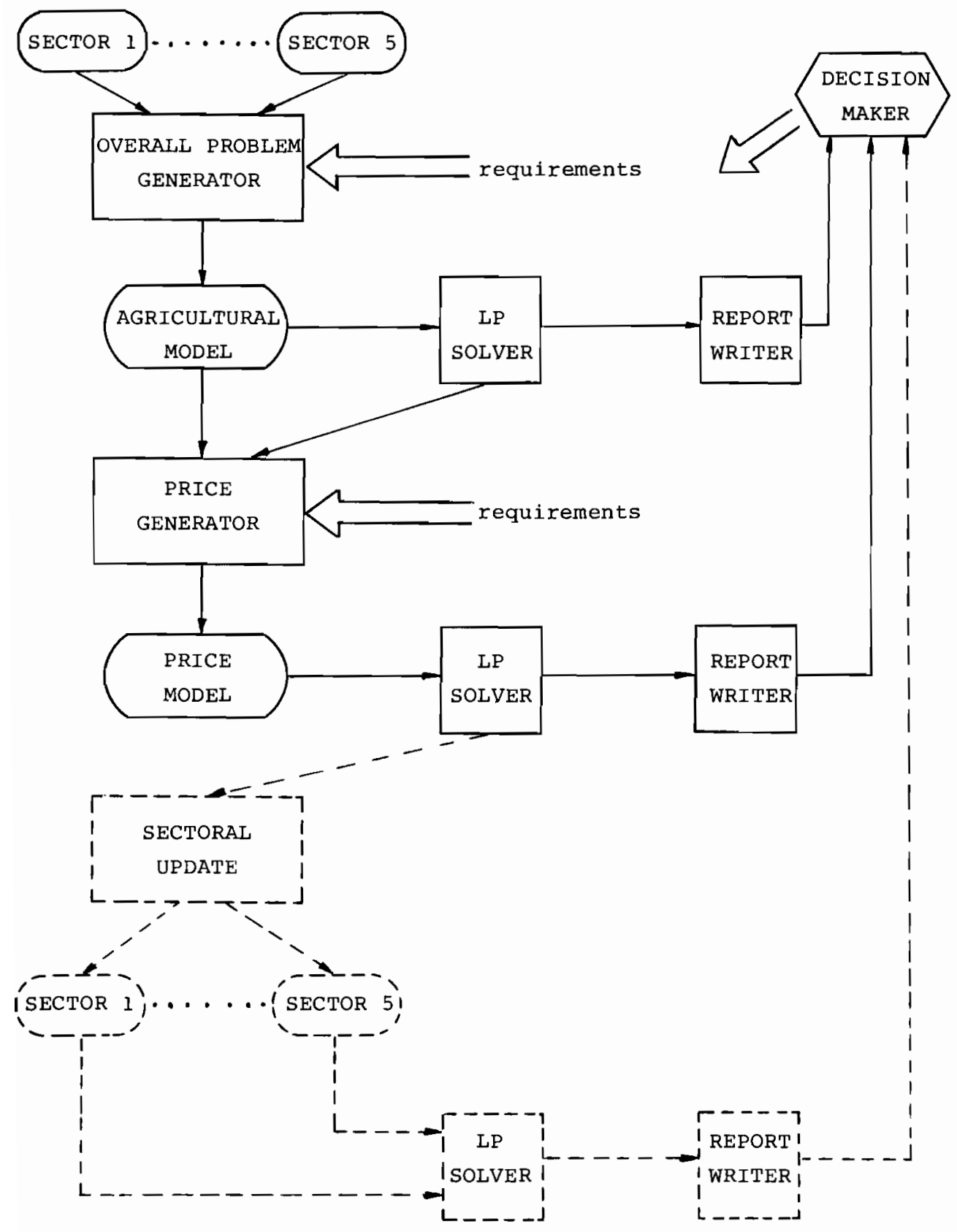

Figure 3. Structure of the decision support system. 


\section{ACKNOWLEDGEMENTS}

The authors thank Prof. A.P. Wierzbicki and Prof. Kirit S. Parikh who have supported the research both by helpful discussions and by arranging cooperation with IIASA. Thanks are also due to Dr. L. Podkaminer for formulating the problem and for many fruitful discussions. Dr. A. Lewandowski's help with regard to multiobjective software is gratefully acknowledged.

\section{REFERENCES}

[1] Polish Agriculture Model. PAM-1, IIASA, forthcoming.

[2] M. Makowski and J. Sosnowski. Coordination of sectoral production planning using prices and quotas (a case study for the Polish agriculture model). CP-81-38, IIASA, 1981.

[3] M. Makowski and J. Sosnowski. Planning and controlling agricultural production in a centrally planned economy with a decentralized decision structure. Research Report, forthcoming.

[4] L. Podkaminer. Efficient use of prices and quantity constraints for control and coordination of linear sectoral production models. WP-81-110, IIASA, 1981.

[5] A.P. Wierzbicki. A methodological guide to multiobjective optimization. WP-79-122, IIASA, 1979.

[6] H. Nikaido. Convex Structures and Economic Theory. Academic Press, 1968.

[7] A. Lewandowski. A program package for linear multiple criteria reference point optimization, short user manual. WP-82-80, IIASA, 1982 .

[8] A.P. Wierzbicki. Private communication, 1980.

[9] M. Makowski and J. Sosnowski. Implementation of an algorithm for scaling matrices and other programs useful in linear programming. CP-81-37, IIASA, 1981. 